\title{
Dosimetric characteristics of a new unit for electronic skin brachytherapy
}

\author{
Teresa Garcia-Martinez', Jan-Pieter Chan², Jose Perez-Calatayud, PhDl,3. Facundo Ballester, PhD4 \\ 'Department of Radiation Oncology, La Fe University and Polytechnic Hospital, Valencia, Spain, 2PANalytical B.V., Eindhoven, the Netherlands, \\ ${ }^{3}$ Department of Radiotherapy, Clinica Benidorm, Benidorm, Spain, ${ }^{4}$ Department of Atomic, Molecular and Nuclear Physics, University of Valencia. \\ Burjassor, Spain
}

\begin{abstract}
Purpose: Brachytherapy with radioactive high dose rate (HDR) ${ }^{192}$ Ir source is applied to small skin cancer lesions, using surface applicators, i.e. Leipzig or Valencia type. New developments in the field of radiotherapy for skin cancer include electronic brachytherapy. This technique involves the placement of an HDR X-ray source close to the skin, therefore combining the benefits of brachytherapy with the reduced shielding requirements and targeted energy of low energy X-rays. Recently, the Esteya ${ }^{\circledR}$ Electronic Brachytherapy System (Esteya EBS, Elekta AB-Nucletron, Stockholm, Sweden) has been developed specifically for HDR brachytherapy treatment of surface lesions. The system provides radionuclide free HDR brachytherapy by means of a small $69.5 \mathrm{kV}$ X-ray source. The purpose of this study is to obtain the dosimetric characterization required for clinical implementation, providing the detailed methodology to perform the commissioning.

Material and methods: Flatness, symmetry and penumbra, percentage of depth dose (PDD), kV stability, HVL, output, spectrum, linearity, and leakage have been evaluated for a set of applicators (from $10 \mathrm{~mm}$ to $30 \mathrm{~mm}$ in diameter).

Results: Flatness and symmetry resulted better than $5 \%$ with around $1 \mathrm{~mm}$ of penumbra. The depth dose gradient is about $7 \% / \mathrm{mm}$. A kV value of $68.4 \pm 1.0 \mathrm{kV}(k=1)$ was obtained, in good agreement with manufacturer data $(69.5 \mathrm{kV})$. HVL was $1.85 \mathrm{~mm}$ Al. Dose rate for a typical $6 \mathrm{~Gy}$ to $7 \mathrm{~Gy}$ prescription resulted about $3.3 \mathrm{~Gy} / \mathrm{min}$ and the leakage value was $<100 \mu \mathrm{Gy} / \mathrm{min}$.

Conclusions: The new Esteya ${ }^{\circledR}$ Electronic Brachytherapy System presents excellent flatness and penumbra as with the Valencia applicator case, combined with an improved PDD, allowing treatment of lesions of up to a depth of $5 \mathrm{~mm}$ in combination with reduced treatment duration. The Esteya unit allows HDR brachytherapy superficial treatment within a minimally shielded environment due its low energy.

Key words: electronic brachytherapy, nonmelanoma skin-cancer, skin brachytherapy, superficial radiotherapy.

\section{Purpose}

Brachytherapy is a radiotherapy modality where a radiation source (a radionuclide or X-ray unit) is placed inside, or close to, the area that requires a treatment. Brachytherapy is widely used for the treatment of skin cancer [1]. Practical advantages of brachytherapy, especially in small superficial lesions, come from its high accuracy and efficiency as a result of dosimetric and set-up aspects. Accurate positioning of radiation source, sharp penumbra, and quick dose fall-off are some of the key benefits of brachytherapy.

Electron beam radiotherapy, which is also often used for skin cancer treatment, requires the use of cut-outs, bolus and collimation, and needs much more time for dosimetric calculations, preparation/verification and treatment deliv- ery. Templates and cut-outs can be time consuming to create and cumbersome to use, since the percentage of depth dose (PDD) and output change according to the specific treatment conditions. Additionally, these customized devices may increase the chance of an error during set-up.

In case of small skin cancer lesions, brachytherapy with a radioactive high dose rate (HDR) ${ }^{192} \mathrm{Ir}$ source is often applied, specially using surface applicators, i.e. Leipzig or Valencia type from Elekta (Elekta AB-Nucletron, Stockholm, Sweden). These applicators allow collimation of the radiation to the area of interest, thereby providing an extremely conformal therapy without unnecessary irradiation of normal healthy tissue. The use of these applicators is limited to flat surfaces and treatment areas up to $3-4.5 \mathrm{~cm}$ in diameter. Typical prescription depth with these applicators is between $3 \mathrm{~mm}$ and $4 \mathrm{~mm}$. Clini- 
cal implementation and dosimetry of skin applicators have been reported elsewhere in the literature [2-10].

New developments in the field of radiotherapy for skin cancer include the introduction of electronic brachytherapy. It involves the placement of an HDR X-ray source positioned directly into skin applicators, combining the benefits of brachytherapy with reduced shielding requirements and targeted energy of low energy X-rays. This offers even more refined approach for skin cancer treatment and further improves conformity of radiation dose to the skin lesion, while sparing surrounding healthy tissues.

Evans et al. [11] made the performance assessment of the Gulmay D3300 kilovoltage (combined superficial and orthovoltage) X-ray therapy unit presenting the key dosimetric beam parameters required for routine patient treatment. Beam half-value layers, the applicator, system interlocks, and dose monitor performance were found to be satisfactory. The difficulties of obtaining percentage of depth dose measurements were discussed.

Jurado et al. [12] evaluated the Therapax SXT 150 unit, which encompass both low and medium-energy beams. The timer error was significant for all filters and should be taken into consideration for absorbed dose rate determination under reference conditions, as well as for the calculation of treatment times. They recommended PDD measurements for each filter-applicator combination. Beam profiles had small penumbras, good symmetry and flatness, except for the lowest energy beam, for which a heel effect was observed. They conclude that the output factor definition of the IAEA TRS-398 protocol for medium-energy $\mathrm{X}$-ray qualities involves the use of data that is difficult to measure.

The model S700 Axxent ${ }^{\mathrm{TM}}$ X-Ray Source (Xoft Inc., San Jose, CA, USA) for electronic brachytherapy was studied by Rivard [13]. This source exhibited depth dose behavior similar to low-energy photon-emitting low dose rate sources ${ }^{125} \mathrm{I}$ and ${ }^{103} \mathrm{Pd}$, yet with capability for variable and much higher dose rates, and subsequently adjustable penetration possibilities. Liu et al. [14] performed the spectral and attenuation curve measurements to characterize it in terms of spectrum and half-value layers. The change in beam quality due to source to source variation and source aging is significant, and each source should be treated on individual basis. Geant4 simulations of the complete X-ray tube in the forward direction yield photon spectra comparable to measured spectra with HVLs matching within uncertainty. However, simulations of the beam in the $90^{\circ}$ direction did not match experiments. The cause for the discrepancy remains unknown. Rong and Welsh [15] provided comprehensive calibration procedures for medical physicists in using the Xoft eBx system and skin applicators.

The Emerging Technology Committee of the American Society for Therapeutic Radiology and Oncology (ASTRO) appointed a Task Group within its Evaluation Subcommittee to evaluate new electronic brachytherapy methods that are being developed for (or are already in) clinical use [16]. The Task Group evaluated two devices, the Axxent Electronic Brachytherapy System by Xoft Inc. (San Jose, CA, USA), and the Intrabeam Photon Radiosur- gery Device by Carl Zeiss Surgical (Oberkochen, Germany). These devices are designed to deliver electronically generated radiation, and because of their relatively low energy output, they do not fall under existing regulatory scrutiny of radioactive sources that are used for conventional radionuclide-based brachytherapy. This report provides a descriptive overview of the technologies, current and future projected applications, comparison of competing technologies, potential impact, and potential safety issues.

Recently, the Esteya ${ }^{\circledR}$ Electronic Brachytherapy System (Esteya EBS, Elekta AB-Nucletron, Stockholm, Sweden) has been developed specifically for HDR brachytherapy treatment of skin lesions. The system provides radionuclide-free HDR brachytherapy by using a miniature $69.5 \mathrm{kV}$ X-ray source. The purpose of this study is to obtain the dosimetric characterization required for clinical implementation, providing the detailed methodology to perform the commissioning.

\section{Material and methods}

\section{Estey $a^{\circledR}$ Electronic Brachytherapy System description}

The Esteya ${ }^{\circledR}$ Electronic Brachytherapy System consists of a treatment unit with surface applicators, a user interface with planning software and a treatment control panel. The Esteya system was designed to obtain dose distributions to the skin surface similar to the dose distributions obtained with the Nucletron Valencia Applicator, a shielded applicator placed directly on the skin surface that, in combination with an ${ }^{192}$ Ir afterloader, provides a focused and uniform dose to surface lesions.

The Esteya treatment unit is portable with four swiveling wheels and an adjustable positioning arm that ease both alignment, and positioning of the X-ray applicator on the patient (Fig. 1). The treatment unit contains the $X$-ray source, associated surface applicator and applicator plastic cap. The treatment unit displays a light field for accurate positioning. The nominal source to surface distance (SSD) is $6 \mathrm{~cm}$.

A set of five surface applicators can be used to modify the size of the X-ray field: $10 \mathrm{~mm}, 15 \mathrm{~mm}, 20 \mathrm{~mm}, 25 \mathrm{~mm}$, and $30 \mathrm{~mm}$ diameter. A clean and (if required) disinfected or sterilized plastic cap is attached to the surface applicator to prevent potential cross-contamination between patients. The surface applicator with plastic applicator cap is placed on the patient skin. There is a special fine tuning mechanism to accurately fit the applicator to the patient surface. The electronic brachytherapy system has a Quality Assurance Device, which provides reproducible daily quality checks (output, flatness and PDD constancy) of the $\mathrm{X}$-ray source and system configuration.

The electronic brachytherapy system has a self-test to check the software and hardware configuration. Carrying out a treatment delivery is possible only when all checks of the self-test have passed successfully, treatment is inhibited if a single check fails. The oil cooling system maintains the temperature of the outside of the X-ray tube, which can be in contact with the patient, below $41^{\circ} \mathrm{C}$. The system controllers associated software checks 
that the correct surface applicator is installed before radiation therapy is initiated. This software controls the fraction delivery parameters, such as treatment duration and dose rate. The X-ray source voltage has a fixed nominal value of $69.5 \mathrm{kV}$. The default current is $1.6 \mathrm{~mA}$, which is automatically adjusted to $1.0 \mathrm{~mA}$ for fractions smaller than $4 \mathrm{~Gy}$, and to $0.5 \mathrm{~mA}$ for fractions smaller than 2 Gy to keep the fraction duration relatively constant and less dependent of the fraction dose. An aluminum flattening filter of $1.6 \mathrm{~mm}$ thick is used to generate a maximum nominal dose rate at zero depth of $3.3 \mathrm{~Gy} / \mathrm{min}$ with a flattened dose profile on the skin.

The computer contains the software for patient administration, treatment planning, treatment delivery, user management, and quality assurance. A treatment planning application is included in the system: after dose per fraction, depth and applicator size are selected, the system presents automatically the required treatment time. It is computed from manufacturer tables of PDD and output. The hard wired control panel is used to start, interrupt and stop a treatment.

\section{Flatness and symmetry evaluation}

Flatness, symmetry and penumbra of all available Esteya surface applicator sizes were evaluated using GafChromic ${ }^{\circledR}$ EBT2 radiochromic films (Grafchromic, ISP, USA) and the EPSON Expression 10000XL Photo flatbed scanner (Seiko Epson Corp., Nagano-Ken, Japan). A set of three radiochromic films were located at $5 \mathrm{~mm}$ depth in plastic water and irradiated for each surface applicator. The depth of $5 \mathrm{~mm}$ was selected, because the application of this unit (according to manufacturer specifications) is for depths up to $5 \mathrm{~mm}$. Consequently, the flatness and penumbra were evaluated at this maximum depth. To consider the response linearity, a film calibration curve with the applicable beam quality was obtained.

The digitalization and evaluation processes were made following our clinical routine protocol: films were digitized prior to irradiation, and $24 \mathrm{~h}$ after the pre-exposure pixel values were subtracted from the post-exposure ones (in a pixel by pixel process). This background subtraction, performed with home-made software, allowed reducing inaccuracies in scan measurements. All film pieces were scanned in transmission mode four consecutive times at a resolution of $100 \mathrm{dpi}$ and 48 bit color. The final stored image was calculated as the average of those four images.

The IEC 60976 [17] criteria was followed for flatness and symmetry, similar to other radiotherapy treatment units. It was applied to the flattened area of each profile (flatness is defined as $80 \%$ of the distance between the two points, where dose takes $50 \%$ of the maximum dose; such a distance is larger than the applicator nominal size) were as follow:

Flatness $=\frac{D_{\max }-D_{\min }}{D_{\max }+D_{\min }}$

where $D_{\max }$ and $D_{\min }$ are the maximum and minimum dose values measured in the flattened area of each profile.

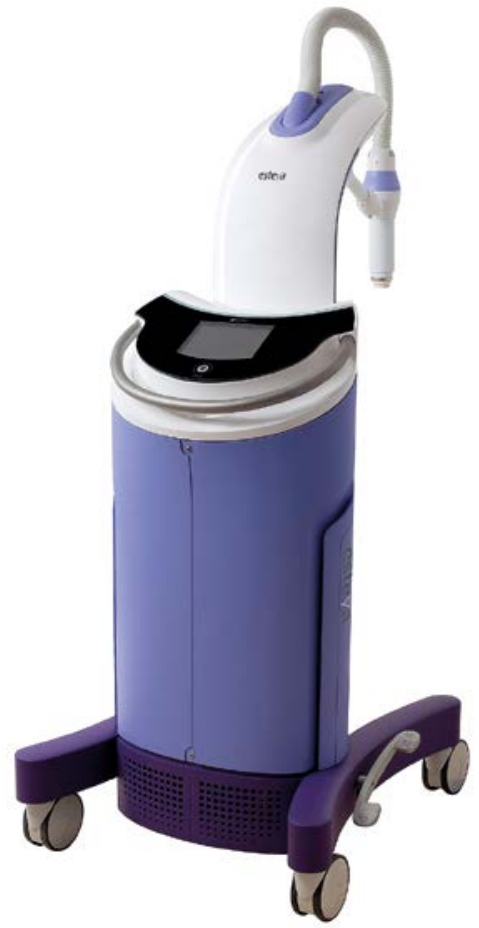

Fig. 1. The Esteya Electronic Brachytherapy System

Symmetry $=\max \left[\frac{D(x)}{D(-x)}\right]$

where $D(x)$ is the dose at point $x ; x$ and $-x$ are points within the flattened region, symmetrical with respect to the central axis. Symmetry as defined as being the maximum ratio within the flattened region.

Penumbra regions were quantified for both $\mathrm{AB}$ and GT profiles as the distance between points with dose values corresponding to the $80 \%$ and $20 \%$ of the dose at the profile center.

\section{Depth dose evaluation}

To perform relative and absolute dose measurements, a soft X-ray parallel-plate chamber PTW T34013 model (PTW, Freiburg, Germany) was used. As indicated by the manufacturer, the collection volume of the chamber is $0.0053 \mathrm{~cm}^{3}$, the entrance window thickness is $0.03 \mathrm{~mm}$, and the effective diameter is $1.7 \mathrm{~mm}$. Because of its small active area, the chamber would be appropriate to measure field sizes as small as $0.5 \mathrm{~cm} \times 0.5 \mathrm{~cm}$.

Plastic water phantom (Plastic Water Low Range, CIRS, Norfolk, VA, USA) was used. This plastic material has a density of $1.03 \mathrm{~g} / \mathrm{cm}^{3}$. The equivalence of this material to liquid water at the energies considered in this work (a spectrum from $11 \mathrm{keV}$ to $70 \mathrm{keV}$; see below) is 0.9781 [18]. The plate parallel chamber was placed in a specific plastic water slab with a groove to accommodate it, so that the chamber surface is flush to the slab surface. This solid water slab was designed specially to carry out the PDD measurement. Plastic water slabs of different thick- 
ness were placed sequentially on top of the chamber, from 0 to $10 \mathrm{~mm}$. Enough solid water slabs were placed below it to ensure full backscatter.

\section{$k V$ stability}

To evaluate the accuracy of the nominal kV (indicated by the device's software as $69.5 \mathrm{kV}$ ) and the reproducibility of the $\mathrm{kV}$ value, the Barracuda multimeter (RTI Electronics AB, Mölndal, Sweden) and its associated software oRTIgo (oRTIgo QA software for Barracuda, Version $6.4 \mathrm{C}$ ) were used.

The oRTIgo software has a large number of templates for different tests: accuracy, reproducibility, half value layer (HVL) and many more. Theses templates checks several parameters, and in the 'Test setup' it is possible to specify acceptance limits and other parameters for use in the evaluation of accuracy and reproducibility. The Pass/ Fail criteria have been defined here.

The Multi-Purpose Detector (MPD) in the Barracuda multimeter is able to measure field sizes less than $3 \mathrm{~mm}$ width and low output levels down to approximately $1 \mu \mathrm{Gy} / \mathrm{s}$. Basically, the detector packages consist of four separate electrometer channels connected to the detectors, with $2.7 \mathrm{~mm}$ wide active section, and a moveable filter package that contains six different filter combinations.

To evaluate the tube voltage accuracy, the measured $\mathrm{kV}$ was compared against the generator set values. The difference between measured and set $\mathrm{kVp}$ was calculated. A Test Setup was configured to check the reproducibility of this X-ray system specifying the maximum allowed deviation: the measured $\mathrm{kVp}$ must fall within a predefined $n \%$ of the selected $\mathrm{kVp}$. The test failed if any exposure fell outside the average $n \%$. The test also failed if the coefficient of variation (ratio between the standard deviation and the mean) exceeded the value we chose in the Test Setup.

\section{HVL evaluation}

HVL measurements were performed according to the AAPM Radiation Therapy Task Group 61 recommendations [19], using the Barracuda system and high purity aluminum slabs (from $0.5 \mathrm{~mm}$ to $2 \mathrm{~mm}$ in thickness). For this measurement, the smaller available applicator (10 $\mathrm{mm}$ in diameter) was used. The set-up follows the TG-61 and TRS-398 [21] recommendations with a source to detect the distance of $56.0 \mathrm{~cm}$, and aluminum-slabs to sense the distance of $50.0 \mathrm{~cm}$. From these measurements, the specific oRTIgo software provides a HVL value. This test requires the Barracuda with the MPD. HVL was calculated using filters on top of the detector. We began with three exposures (without any aluminum filter added) to get the zero-point and to check the consistency of the generator. We added the aluminum filters and recorded the readings. A measurement was made for each thickness and the mean value was used.

There are several detectors inside the MPD and the total filtration is determined from a combination of these signals and the $\mathrm{kV}$ value. The software determined the total filtration dynamically, which means that if an extra filtration is added during a measurement, the software notices the change.
As soon as the measured dose was reduced to $50 \%$ of the no-filter value, the HVL was calculated. One exposure must be made without filter, and at least one exposure close to either side of the $50 \%$ value.

The HVL was computed using the following formula:

$$
H V L=T_{b} \ln \left[\frac{2 E_{a}}{E_{o}}\right]-\frac{T_{a} \ln \left[\frac{2 E_{b}}{E_{o}}\right]}{\operatorname{In}\left[\frac{E_{a}}{E_{b}}\right]}
$$

where Eo is the direct exposure reading without test filtration, Ea - exposure reading above $50 \%$, Eb - exposure reading below $50 \%$, Ta - test filtration used when Ea measurement was made, $\mathrm{Tb}$ - test filtration used when $\mathrm{Eb}$ measurement was made.

The uncertainty in estimating total filtration between $60 \mathrm{kV}$ and $120 \mathrm{kV}$ is (according to the Barracuda manual) $\pm 10 \% \mathrm{~mm} \mathrm{Al}$ or $\pm 0.3 \mathrm{~mm} \mathrm{Al}(k=2)$, respectively. In addition, independently of the Ortigo method, a fit of the readings, according to the TG-61 and TRS-398 protocols was done to compare the results.

\section{Absolute dose evaluation}

For this evaluation, the PTW parallel-plate chamber T34013 model in combination with the PTW Unidos electrometer on the plastic water phantom was used. Because this chamber is calibrated in absorbed dose to water, $N_{D, W}$, the TRS-398 code of practice for low-energy $\mathrm{kV}$ $\mathrm{X}$-rays beams was followed.

The dose rate at the surface $(0 \mathrm{~mm}$ depth $)$ was determined according to:

$$
D_{w Q}=M_{Q} N_{D, W, Q_{o}} K_{Q Q_{o}}
$$

where $M_{Q}$ is the reading of the dosimeter with the reference point of the chamber positioned at the reference point $z_{r e f}$ (phantom surface) and corrected for temperature and pressure. $N_{D, W, Q_{0}}$ is the calibration factor in terms of absorbed dose to water for the dosimeter at the reference quality $Q_{0}$, and $K_{Q_{0}}$ is a chamber-specific factor, which corrects the differences between the reference beam quality $Q_{0}$ and the actual beam quality being used $Q$.

For the PTW parallel-plate chamber T34013 model used in this work, the $N_{D, W}, Q_{0}$ was $4.887 \times 10^{9} \mathrm{~Gy} \mathrm{C}^{-1} \pm 3.4 \%$ $(k=2)$. This factor was obtained from an accredited Laboratory (PTW Laboratory, Braunschweig, Germany). The calibration is traceable to national standards of the PTB Laboratory (Germany).

$K_{Q_{0}}$ was obtained from the calibration certificate of the PTW. A set of correction factors (ranging from 0.997 to 1.017 ) relative to the quality of the calibration (HVL $0.43 \mathrm{~mm} \mathrm{Al}$ ) for a quality range from $0.10 \mathrm{~mm}$ to $3.10 \mathrm{~mm}$ $\mathrm{Al}$ are included.

Finally, dose rate at $0 \mathrm{~mm}$ depth has been determined from the ratio between $D_{w Q}$ and the measurement time, which is the nominal time in the system console. 
(c) Pinhole 2

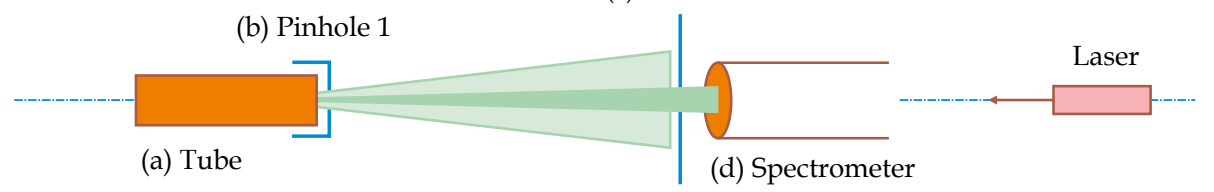

Fig. 2. Schematic view of the spectrum measurement setup

\section{Spectral evaluation}

During tube calibration, the output spectrums were measured (PANalytical B.V., Eindhoven, The Netherlands). The measurement setup consists of the following components (Fig. 2): (a) tube with $30 \mathrm{~mm}$ diameter surface applicator; (b) lead pinhole, thickness $4.5 \mathrm{~mm}$, opening $4 \mathrm{~mm}$ in diameter; (c) lead pinhole, thickness $3 \mathrm{~mm}$, opening $0.7 \mathrm{~mm}$ in diameter; (d) germanium crystal spectrometer Ortec GLP 25300/13P4; diameter 25 mm, thickness $13 \mathrm{~mm}$; beryllium window, thickness $0.25 \mathrm{~mm}$; cooled by liquid nitrogen.

All components were aligned with a laser beam, which represents the central axis. The distance from tube to spectrometer was approximately $1 \mathrm{~m}$. Pinhole 1 is used to reduce scatter radiation and was positioned against the tube, pinhole 2 is used to narrow the line of sight of the spectrometer to the central axis and was placed as close as possible to the spectrometer.

The spectrometer was calibrated by putting a piece of solder (lead and tin) in the beam path. The characteristic lines are fitted with their corresponding energies: $\mathrm{Pb}$ L- $\alpha$ : $10.550 \mathrm{keV}$; Pb L- $\beta$ : $12.612 \mathrm{keV}$; Sn K- $\alpha$ : $25.191 \mathrm{keV;}$ Sn K- $\beta$ : $28.481 \mathrm{keV}$. Also, the endpoint of the energy spectrum $69.5 \mathrm{keV}$ was used. The measurement time of the spectrometer was set to $1000 \mathrm{~s}$. The measured (raw) spectrum was de-convoluted using a Monte Carlo calculated response of the detector. The Penelope2008 Monte Carlo code [20] and the SpekCalc software [22-24] were also used to obtain theoretical spectra to be compared with the measured ones. Penelope2008 is a MC code, which reliability and performance have been widely tested [25]. It employs a mixed procedure to simulate electron and positron interactions (elastic scattering, inelastic scattering and bremsstrahlung emission), in which 'hard' events (i.e. those with deflection angle and/or energy loss larger than pre-selected cut-offs) are simulated in a detailed way, while 'soft' interactions are calculated from multiple scattering approaches. Photon interactions (Rayleigh scattering, Compton scattering, photoelectric effect, and electron-positron pair production) and positron annihilation are simulated in a detailed way.

Penelope2008 cross section data are those of the EPDL97 [26] and EEDL [27], except that for Compton interactions Penelope2008 uses the impulse approximation to account for Doppler broadening, and binding effects in the photon and electron cross-sections.

The X-ray source was modelled according to the characteristics specified by the manufacturer. Source spectra were calculated striking $69.5 \mathrm{keV}$ mono-energetic electrons in the tungsten anode surface to generate X-rays.
The cut-off energy for electrons and photons was considered $1 \mathrm{keV}$. Spectrum was binned at $0.5 \mathrm{keV}$ intervals. $10^{9}$ electron histories were generated to produce $k=1$ Type A uncertainties below $1 \%$.

\section{Linearity}

As described above, the system changes the intensity according to prescribed dose and depth, in order to reduce the treatment time. To verify the linearity of this conversion, ionization measurements with the T34013 chamber on the plastic water phantom using the $30 \mathrm{~mm}$ applicator have been done at different nominal prescribed dose, evaluating the collected charge versus the $\mathrm{mA} \times \mathrm{s}$ settings.

\section{Leakage estimation evaluation}

A home-made setup was prepared to enable leakage measurements (Fig. 3). A $3 \mathrm{~mm}$ thick lead slab was used to block the primary beam. Leakage was measured at four points at the level of the applicator surface $(10 \mathrm{~mm}$ applicator, since it is the smallest field size available) and as close as possible to the X-ray tube. Additionally, one extra measurement at a height of $60 \mathrm{~mm}$ was also made.

To have a good spatial resolution, the PTW T34013 chamber (the same that was used for absolute dose and PDD measurements) was used. Despite the harder spectrum, we assume that the calibration factor of this chamber is adequate, because of very small resulted value and upper limit obtained. Strictly speaking, due to the harder spectrum, the calibration factor might change. However, since this is an upper limit and the value obtained is very small, we consider that any reasonable modification in the calibration factor will not play a significant role.

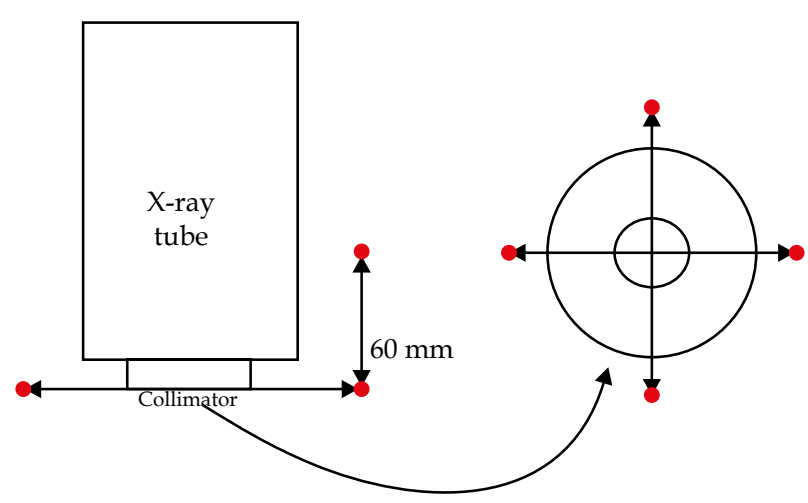

Fig. 3. Schematic representation of the setup for the leakage evaluation 
The detected signal for the chamber is very low, so pre-irradiation and post-irradiation chamber leakage were estimated and taken into account to correct the device leakage measurement during irradiation.

\section{Results}

\section{Flatness and symmetry evaluation}

Table 1 presents the mean flatness and symmetry and Table 2 shows the penumbra for each applicator size, along the two main axes (along the gantry-target direction [GT] and lateral or perpendicular to the gantry-target direction $[\mathrm{AB}])$. Typically, flatness below $5 \%$ and symmetry in the interval $95 \%$ to $105 \%$ are fulfilled for all applicators sizes, keeping the penumbra about $1 \mathrm{~mm}$; higher dose values are obtained on the $\mathrm{G}$ direction vs. $\mathrm{T}$ and on the $\mathrm{B}$ direction vs. A. Since there is a small angular deviation between anode and cathode, a corresponding deviation on the electron beam appears, and therefore a slight "heel effect". Such heel effect causes an asymmetry of $1.5 \%$ of the beam profile in one direction (GT), which contributes to the deviation in the flatness of $4.3 \%$. Without the heel effect, the flatness deviation is $2.8 \%$ on average. Values of penumbra on both axes are basically the same. Estimated uncertainties $(k=1)$ in flatness and symmetry have been approximately $1 \%$, while for the case of the penumbra it was lower than $0.1 \mathrm{~mm}$.

\section{Depth dose evaluation}

Table 3 presents the PDD data normalized at maximum (0 $\mathrm{mm}$ depth) up to a depth of $10 \mathrm{~mm}$. The standard devi- ation of the PDD measurements was $0.25 \%(k=1)$. As expected, we observed a slight increment of PDD with surface applicator diameter. At the typical prescription depth of $3 \mathrm{~mm}$, the range resulted about $1 \%$, reaching $4 \%$ at the maximum depth of $10 \mathrm{~mm}$. The dose depth gradient was about $7 \%$ per millimeter. When normalized at the typical prescription depth of $3 \mathrm{~mm}$, the surface dose was $23 \%$ higher.

\section{kV stability}

The $\mathrm{kV}$ mean value obtained by measuring using the Barracuda system and oRTIgo software with accuracy of $\pm 1.5 \%$ (according to the Barracuda manual) was 68.4 $\pm 1.0 \mathrm{kV}(k=1)$, which is compatible with the nominal value of $69.5 \mathrm{kV}$ as specified in the Esteya unit.

\section{HVL evaluation}

The HVL evaluation results from both the oRTIgo calculation and the fit for the $10 \mathrm{~mm}$ surface applicator size were $1.86 \pm 0.20 \mathrm{~mm} \mathrm{Al}$, and $1.82 \pm 0.16 \mathrm{~mm} \mathrm{Al}(k=1)$, respectively. As it can be observed, the results given by oRTIgo software are in good agreement with the data obtained by the negative exponential fit.

\section{Absolute dose evaluation}

The measured absolute-rate values for all the available surface applicators at $0 \mathrm{~mm}$ depth are shown in Table 4 . The output range within applicators was $10 \%$. Treatment time for a typical prescription at $3 \mathrm{~mm}$ depth with the $30 \mathrm{~mm}$ diameter applicator of 6 Gy to 7 Gy per fraction gives $109 \mathrm{~s}$ to $127 \mathrm{~s}$. Dose rate decreases with applicator size.

Table 1. Flatness and symmetry results of the Esteya unit results for all available surface applicators. Uncertainties are given with a coverage factor $k=1$

\begin{tabular}{ccccc} 
Surface applicator & \multicolumn{2}{c}{ Flatness } & \multicolumn{2}{c}{ Symmetry } \\
\cline { 2 - 5 } diameter $[\mathrm{mm}]$ & $\mathrm{GT}$ & $\mathrm{AB}$ & $\mathrm{GT}$ & $\mathrm{AB}$ \\
\hline 30 & $4.3 \pm 0.3 \%$ & $2.8 \pm 0.1 \%$ & $104.8 \pm 0.5 \%$ & $96.6 \pm 0.3 \%$ \\
\hline 25 & $3.7 \pm 0.8 \%$ & $3.0 \pm 2.3 \%$ & $104.6 \pm 0.6 \%$ & $94.1 \pm 2.7 \%$ \\
\hline 20 & $3.4 \pm 0.6 \%$ & $3.5 \pm 0.5 \%$ & $103.3 \pm 0.8 \%$ & $97.2 \pm 0.8 \%$ \\
\hline 15 & $4.4 \pm 0.1 \%$ & $4.6 \pm 1.2 \%$ & $103.4 \pm 0.4 \%$ & $95.9 \pm 3.1 \%$ \\
\hline 10 & $6.9 \pm 1.0 \%$ & $7.4 \pm 1.6 \%$ & $106.0 \pm 1.4 \%$ & $99.0 \pm 9.5 \%$ \\
\hline
\end{tabular}

Table 2. Penumbra evaluation results for all available surface applicators. Uncertainties are given with a coverage factor $k=1$

Surface applicator diameter [mm] Direction penumbra $[\mathrm{mm}]$

\begin{tabular}{ccccc} 
diameter $[\mathrm{mm}]$ & $\mathrm{G}$ & $\mathrm{T}$ & $\mathrm{A}$ & $\mathrm{B}$ \\
\hline 30 & $0.88 \pm 0.02$ & $0.98 \pm 0.04$ & $1.10 \pm 0.09$ & $1.14 \pm 0.07$ \\
\hline 25 & $1.15 \pm 0.01$ & $1.25 \pm 0.04$ & $1.30 \pm 0.01$ & $1.31 \pm 0.01$ \\
\hline 20 & $1.04 \pm 0.06$ & $1.10 \pm 0.05$ & $1.27 \pm 0.04$ & $1.22 \pm 0.03$ \\
\hline 15 & $0.97 \pm 0.03$ & $1.05 \pm 0.06$ & $1.12 \pm 0.15$ & $1.12 \pm 0.02$ \\
\hline 10 & $0.86 \pm 0.01$ & $0.87 \pm 0.18$ & $1.06 \pm 0.10$ & $0.99 \pm 0.15$ \\
\hline
\end{tabular}


Table 3. PDD data for each surface applicator size normalized to the maximum value at $0 \mathrm{~mm}$

\begin{tabular}{cccccc}
\multirow{2}{*}{ Depth $[\mathrm{mm}]$} & \multicolumn{5}{c}{ Surface applicator diameter $[\mathrm{mm}]$} \\
\cline { 2 - 5 } & 10 & 15 & 20 & 25 & 30 \\
\hline 0 & $100.0 \%$ & $100.0 \%$ & $100.0 \%$ & $100.0 \%$ & $100.0 \%$ \\
\hline 1 & $92.7 \%$ & $92.3 \%$ & $92.4 \%$ & $92.4 \%$ & $92.5 \%$ \\
\hline 2 & $87.6 \%$ & $85.9 \%$ & $86.2 \%$ & $86.3 \%$ & $86.5 \%$ \\
\hline 3 & $80.1 \%$ & $80.6 \%$ & $80.4 \%$ & $81.0 \%$ & $81.0 \%$ \\
\hline 4 & $73.1 \%$ & $74.4 \%$ & $74.6 \%$ & $75.5 \%$ & $75.8 \%$ \\
\hline 5 & $68.6 \%$ & $69.9 \%$ & $70.9 \%$ & $71.3 \%$ & $71.7 \%$ \\
\hline 6 & $63.8 \%$ & $65.0 \%$ & $65.8 \%$ & $67.0 \%$ & $67.4 \%$ \\
\hline 7 & $59.1 \%$ & $60.7 \%$ & $61.8 \%$ & $62.6 \%$ & $62.9 \%$ \\
\hline 8 & $55.9 \%$ & $57.0 \%$ & $57.9 \%$ & $58.4 \%$ & $59.0 \%$ \\
\hline 9 & $51.7 \%$ & $52.5 \%$ & $54.3 \%$ & $55.1 \%$ & $55.3 \%$ \\
\hline 10 & $48.5 \%$ & $50.0 \%$ & $51.3 \%$ & $52.2 \%$ & $52.7 \%$ \\
\hline
\end{tabular}

\section{Spectral evaluation}

The spectra of six different tubes have been measured. Their average energy is $36.2 \pm 0.1 \mathrm{keV},(k=1)$. Figure 4 shows the Esteya system measured spectrum compared with the spectra obtained with Penelope (average energy $36.2 \mathrm{keV}$ ) and SpekCalc (average energy $36.6 \mathrm{keV}$ ).

\section{Linearity}

Linearity of the Esteya EBS was checked for absorbed doses between $1 \mathrm{~Gy}$ and $6 \mathrm{~Gy}$, which implies an interval of $80 \mathrm{~s}$ to $131 \mathrm{~s}$, and intensity of $0.5 \mathrm{~mA}$ to $1.6 \mathrm{~mA}$. A linear fit of the results yielded a correlation coefficient $R^{2}=0.994$. So, the system presents an adequate linearity along a wide dose range for which it has been designed. It agrees with manufacturer specification: "The timing of the X-ray beam is controlled by the primary timer, and is checked by an independent secondary timer, manufacturer guarantees that timing accuracy is within $1 \% "$.

\section{Leakage estimation evaluation}

In the most adverse situation, the corrected leakage value obtained was smaller than $2 \mu \mathrm{Gy} / \mathrm{s}$. Taking into account the dose rate measured for the used surface applicator, this leakage value would be less than $0.01 \%$ of the delivered dose. These values can be considered as an upper threshold, concluding that the system has negligible leakage.

\section{Uncertainty analysis}

$D_{w Q}$ uncertainties have been evaluated, taken into account that the product $N_{D, W, Q_{0}} K_{Q_{Q}}$ has an uncertainty of $3.3 \%(k=2)$ and $M_{Q}$ of $0.25 \%(k=1)$, it results in a uncertainty of $1.7 \%(k=1)$.
Table 4. Absolute dose rate values and its standard deviation $(k=1)$ at $0 \mathrm{~mm}$ depth for the five surface applicators

\begin{tabular}{cc} 
Applicator diameter $[\mathrm{mm}]$ & $\begin{array}{c}\text { Dose rate at } 0 \mathrm{~mm} \\
\text { depth } D_{w}\left(\mathrm{~Gy} \mathrm{~min}^{-1}\right)\end{array}$ \\
\hline 10 & $3.004 \pm 0.013$ \\
\hline 15 & $3.122 \pm 0.001$ \\
\hline 20 & $3.197 \pm 0.002$ \\
\hline 25 & $3.300 \pm 0.001$ \\
\hline 30 & $3.295 \pm 0.001$ \\
\hline
\end{tabular}

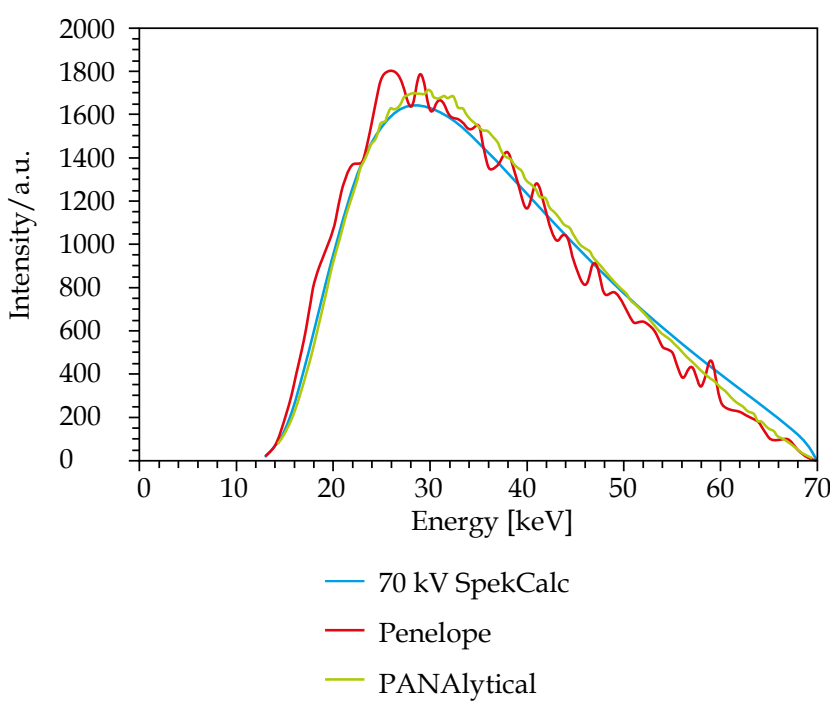

Fig. 4. Esteya electronic brachytherapy system spectrum 


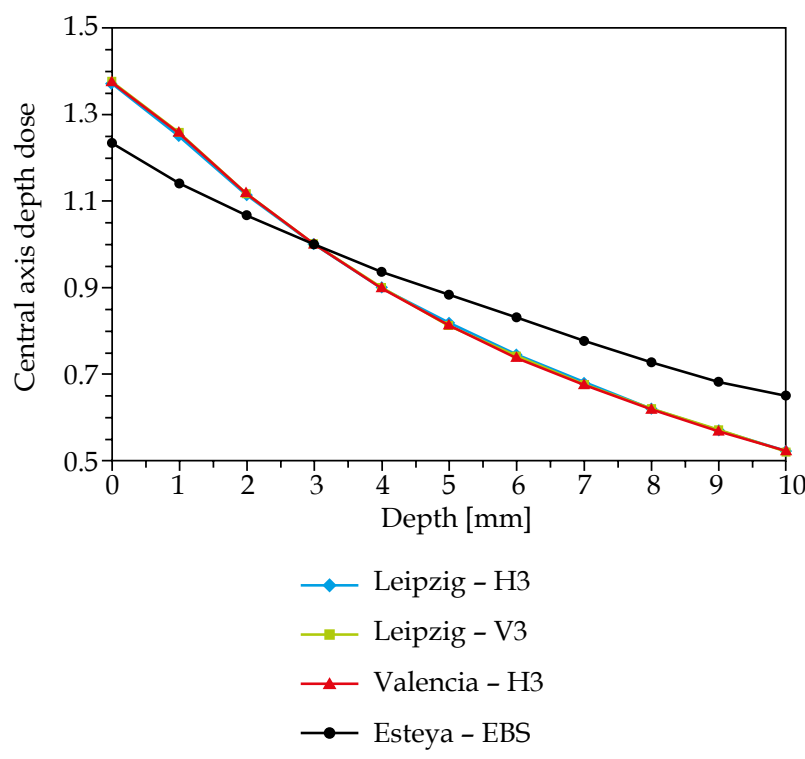

Fig. $5.30 \mathrm{~mm}$ applicator central axis depth dose normalized @ $3 \mathrm{~mm}$

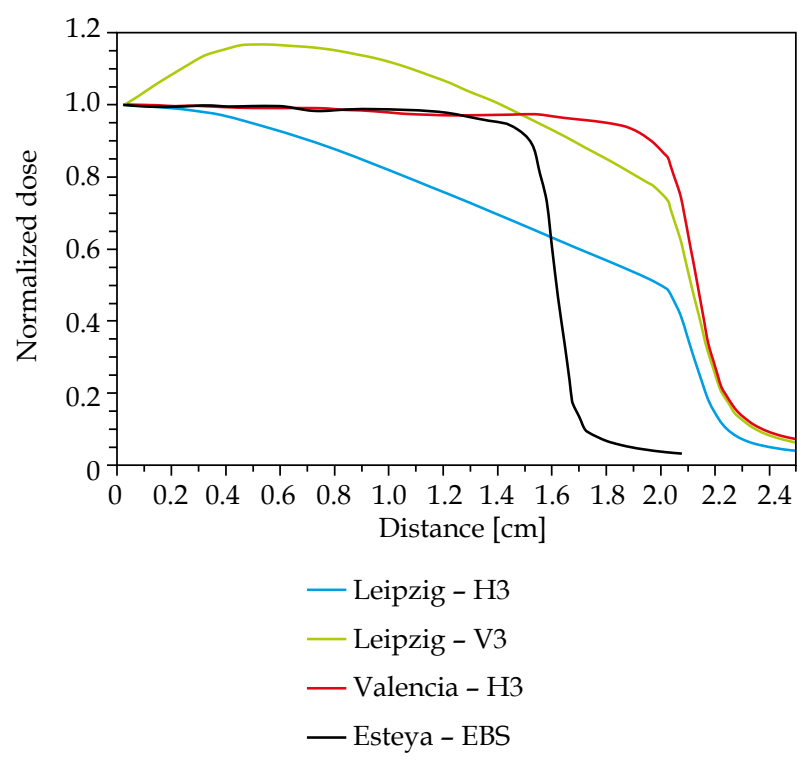

Fig. 6. Half profiles at $5 \mathrm{~mm}$ depth normalized to the central axis value for the $30 \mathrm{~mm}$ in diameter applicators

\section{Discussion}

In Figure 5 we show a comparison of the $30 \mathrm{~mm}$ in diameter applicator - PDD curves for the two Leipzig versions (horizontal and vertical) from Nucletron [3], the Valencia applicator [2], and the results in this work for the Esteya electronic brachytherapy system. Up to the prescription depth, the PDD is improved with a lower surface dose. As a consequence, deeper tissues receive a higher dose. In Figure 6, dose profiles for the $30 \mathrm{~mm}$ in diameter applicators are compared, showing the high homogeneity obtained with the Esteya system, even improving the Valencia's profile. The (80-20\%) penumbra values are significantly improved from Leipzig $(6.2-9.5 \mathrm{~mm})$ to Valencia $(1.9 \mathrm{~mm})$ and Esteya $(1.1 \mathrm{~mm})$.

\section{Conclusions}

Commissioning and dosimetric characteristics of the Esteya system are presented. The new Esteya system of electronic brachytherapy shows excellent flatness and penumbra as in the Valencia applicator case, but with an improved PDD (allowing treatment of lesions up to $5 \mathrm{~mm}$ deep) and increased dose rate (reducing the treatment time). Because of the low energy of Esteya system, it allows HDR brachytherapy superficial treatment within a minimally shielded environment.

\section{Acknowledgements}

This study was supported in part by Generalitat Valenciana (Project PROMETEO2008/114), Spanish Government (Project No. FIS2010-17007), and Nucletron, an Elekta company.

\section{Disclosure}

Authors report no conflict of interest.

\section{References}

1. Guix B, Finestres F, Tello J et al. Treatment of skin carcinomas of the face by high-dose-rate brachytherapy and custom-made surface molds. Int J Rad Onc Biol Phys 2000; 47: 95-102.

2. Granero D, Perez-Calatayud J, Gimeno J et al. Design and evaluation of a HDR skin applicator with flattening filter. Med Phys 2008; 35: 495-503.

3. Perez-Calatayud J, Granero D, Ballester F et al. A dosimetric study of Leipzig applicators. Int J Rad Onc Biol Phys 2005; 62: 579-584.

4. Niu H, Hsi WC, Chu JC et al. Dosimetric characteristics of the Leipzig surface applicators used in the high dose rate brachy radiotherapy. Med Phys 2004; 31: 3372-3377.

5. Evans MD, Yassa M, Podgorsak EB et al. Surface applicators for high dose rate brachytherapy in AIDS-related Kaposi's sarcoma. Int J Rad Onc Biol Phys 1997; 39: 769-774.

6. Pérez-Calatayud J, Granero D, Ballester F et al. Technique for routine output verification of Leipzig applicators with a well chamber. Med Phys 2006; 33: 16-20.

7. Pérez-Calatayud J, Granero D, Ballester F et al. Erratum: "Technique for routine output verification of Leipzig applicators with a well chamber" [Med. Phys. 33, 16-20, 2006]. Med Phys 2006; 33: 2654.

8. Fulkerson RK. Dosimetric characterization of surface applicators for use with high dose rate Ir-192 and electronic brachytherapy sources. PhD dissertation 2012. University of Wisconsin-Madison; p. 165.

9. Fulkerson RK, Micka JA, DeWerd LA. Dosimetric characterization and output verification for conical brachytherapy surface applicators (Part 1: Electronic brachytherapy source). Med Phys 2014; 41; http://dx.doi.org/10.1118/1.4862505.

10. Fulkerson RK, Micka JA, DeWerd LA. Dosimetric characterization and output verification for conical brachytherapy surface applicators (Part 2: High Dose Rate ${ }^{192}$ Ir sources). Med Phys 2014; 41; http://dx.doi.org/10.1118/1.4862506.

11. Evans PA, Moloney AJ, Mountford PJ. Performance assessment of the Gulmay D3300 kilovoltage X-ray therapy unit. $\mathrm{Br}$ J Radiol 2001; 74: 537-547.

12. Jurado D, Eudaldo T, Carrasco P et al. Pantak Therapax SXT 150: performance assessment and dose determination using IAEA TRS-398 protocol. Br J Radiol 2005; 78: 721-732. 
13. Rivard MJ, Davis SD, DeWerd LA et al. Calculated and measured brachytherapy dosimetry parameters in water for the Xoft Axxent X-Ray Source: an electronic brachytherapy source. Med Phys 2006; 33: 4020-4032.

14. Liu D, Poon M, Bazalova M et al. Spectroscopic characterization of a novel electronic brachytherapy system. Phys Med Biol 2008; 53: 61-75.

15. Rong Y, Welsh JS. Surface applicator calibration and commissioning of an electronic brachytherapy system for nonmelanoma skin cancer treatment. Med Phys 2010; 37: 5509-5517.

16. Park CC, Yon MS, Podgorsak MB et al. American Society for Therapeutic Radiology and Oncology (ASTRO) Emerging Technology Committee report on electronic brachytherapy. Int J Rad Onc Biol Phys 2010; 76: 963-972.

17. IEC 60976 report, Medical electrical equipment - Medical electron accelerators - Functional performance characteristics, 2007-10, International Electrotechnical Commission.

18. Ramaseshan R, Kholi K, Cao F et al. Dosimetric evaluation of Plastic Water Diagnostic-Therapy. J Appl Clin Med Phys 2008; 9: 2761.

19. Ma CM, Coffey CW, DeWerd LA et al. AAPM protocol for 40-300 kV x-ray beam dosimetry in radiotherapy and radiobiology. Med Phys 2001; 28: 868-893.

20. Salvat F, Fernández-Varea JM, Sempau J. PENELOPE-2008 A code system for Monte Carlo simulation of electron and photon transport, OECD-NEA, Issy-les-Moulineaux, France. Available from http://www.nea.fr

21. Andreo P, Burns DT, Hohlfeld $\mathrm{K}$ et al. Absorbed dose determination in external beam radiotherapy: an international Code of Practice for dosimetry on standards of absorbed dose to water, in Technical Reports Series No. 398, 2000 IAEA. Available from http:/ / www-pub.iaea.org/books/

22. Poludniowski GG. Calculation of x-ray spectra emerging from an x-ray tube. Part II. X-ray production and filtration in x-ray targets. Med Phys 2007; 34: 2175-2186.

23. Poludniowski GG, Evans PM. Calculation of x-ray spectra emerging from an $\mathrm{x}$-ray tube. Part I. Electron penetration characteristics in x-ray targets. Med Phys 2007; 34: 2164-2174.

24. Poludniowski G, Landry G, DeBlois F et al. SpekCalc: a program to calculate photon spectra from tungsten anode x-ray tubes. Phys Med Biol 2009; 54: 433-438.

25. Ye SJ, Brezovich IA, Pareek P et al. Benchmark of PENELOPE code for low-energy photon transport: dose comparisons with MCNP4 and EGS4. Phys Med Biol 2004; 49: 387-397.

26. Cullen DE, Hubbell JH, Kissel L. EPDL97: the Evaluated Photon Data Library, '97 Version. University of California, Lawrence Livermore National Laboratory, Livermore 1997.

27. Cullen DE, Perkins ST, Seltzer SM. Tables and graphs of electron-interaction cross sections from $10 \mathrm{eV}$ to $100 \mathrm{GeV}$ derived from the LLNL Evaluated Electron Data Library (EEDL), $\mathrm{Z}=1$-100. Lawrence Livermore National Laboratory, Livermore 2001. 